\title{
Impulsivität und assoziierte Konstrukte bei drogenabhängigen Patienten
}

\author{
Daniela Reichl ${ }^{10}$, Elisabeth Bonn¹, Niklas Enewoldsen¹, Stefanie Schwenzner ${ }^{2}$ und \\ Sabine Steins-Loeber ${ }^{1}$ \\ Lehrstuhl für Klinische Psychologie und Psychotherapie, Otto-Friedrich-Universität Bamberg \\ Fachklinik Aggerblick, Overath
}

\begin{abstract}
Zusammenfassung: Zielsetzung: Die vorliegende Pilotstudie untersuchte, ob selbstberichtete Achtsamkeit bei drogenabhängigen Patienten negativ mit impulsivem Verhalten assoziiert ist und ob dieser Zusammenhang über eine effektivere Emotionsregulation vermittelt wird. Ferner wurde die Relevanz der Differenzierung verschiedener Impulsivitäts-Facetten im Zusammenhang mit Craving und Abstinenzzuversicht untersucht. Methodik: Einunddreißig männliche drogenabhängige Patienten in stationärer Entwöhnung beantworteten Fragen zu Achtsamkeit (MAAS), Emotionsregulationskompetenzen (SEK-27), Depressivität (PHQ-9), Craving (MaCS) und Abstinenzzuversicht (HEISA-16). Impulsivität wurde zum einen als selbstberichtetes Trait-Merkmal (BIS-15) erfasst. Weiterhin wurden die verhaltensbezogenen Komponenten impulsives Entscheidungsverhalten (Delay Discouting Task) und substanzbezogene Inhibitionskontrolle (Go-/No-Go-Aufgabe) erhoben. Ergebnisse: Achtsamkeit war negativ mit Trait-Impulsivität assoziiert und hing, mediiert über höhere Emotionsregulationskompetenzen, positiv mit Inhibitionskontrolle zusammen. Trait-Impulsivität und Delay Discounting erwiesen sich als Korrelate von Craving. Keine der Impulsivitäts-Facetten war mit Abstinenzzuversicht assoziiert. Schlussfolgerungen: Die Differenzierung der Impulsivitäts-Facetten stellte sich als bedeutsam heraus. Experimentelle Designs sind nun notwendig, um die in der vorliegenden Pilotstudie gefundenen Zusammenhänge hinsichtlich ihrer Kausalität zu prüfen.
\end{abstract}

Schlüsselwörter: Impulsivität, Craving, Emotionsregulation, Achtsamkeit, Drogenabhängigkeit

\section{Impulsivity and Associated Constructs in Drug Addicted Patients}

\begin{abstract}
Aim: The current pilot study examined, if self-reported mindfulness is negatively associated with impulsive behaviour in drug addicted patients, and if this relationship is mediated by more effective emotion regulation. Furthermore, the relevance of differentiating between various facets of impulsivity in relation to craving and abstinence self-efficacy was investigated. Methods: Thirty-one male drug addicted inpatients answered questions on mindfulness (MAAS), emotion regulation competencies (SEK-27), depression (PHQ-9), craving (MaCS) and abstinence self-efficacy (HEISA-16). Impulsivity was assessed as a self-reported trait (BIS-15). In addition, the behavioural components of impulsive choice (Delay Discounting Task) and substance-related inhibitory control (Go-/No-Go Task) were examined. Results: Mindfulness was negatively associated with trait impulsivity, and - mediated by higher emotion regulation competencies - positively related to inhibitory control. Trait impulsivity and impulsive choice correlated with craving. None of the facets of impulsivity were associated with abstinence self-efficacy. Conclusions: Differentiating between the various facets of impulsivity proved to be important. Experimental designs are now necessary to examine the causal relationships among the associations identified in this pilot study.
\end{abstract}

Keywords: Impulsivity, craving, emotion regulation, mindfulness, drug addiction

\section{Einführung}

Für die Vorhersage von Rückfällen bei Drogenabhängigkeit scheint insbesondere Impulsivität ein wichtiger Prädiktor und therapeutischer Ansatzpunkt zu sein (Schultz, Neumann \& Steins-Loeber, 2019).

Darunter kann die Tendenz zu schnellen, ungeplanten Reaktionen auf internale und externale Reize ohne Berücksichtigung der negativen Konsequenzen für sich und andere verstanden werden (Moeller, Barratt, Dougherty,
Schmitz, \& Swann, 2001, S.1784). Es handelt sich dabei um ein mehrdimensionales Konstrukt (MacKillop et al., 2016). Häufig werden dispositionelles, impulsives Verhalten im Alltag (Trait-Impulsivität), defizitäre Inhibitionskontrolle und impulsives Entscheidungsverhalten unterschieden (z.B. MacKillop et al., 2016). Inhibitionskontrolle bezeichnet dabei die Fähigkeit, dominante motorische Reaktionen zu hemmen. Impulsives Entscheidungsverhalten spiegelt sich z.B. in der Abwertung verzögerter Belohnung wieder (Delay Discounting). 
Trait-Impulsivität scheint bei einer Abhängigkeit von Opioiden (Robles, Huang, Simpson \& McMillan, 2011), Kokain (Coffey, Gudleski, Saladin \& Brady, 2003; Fernández-Serrano, Perales, Moreno-López, Pérez-García \& Verdejo-García, 2012; Verdejo-García, Bechara, Recknor \& Pérez-García, 2006) und insbesondere Stimulanzien (Mohammadzadeh, Khosravani \& Feizi, 2018; Taylor et al., 2016) erhöht zu sein. Weiterhin finden sich bei Drogenabhängigkeit Defizite in der Inhibitionskontrolle (Fernández-Serrano et al., 2012; Verdejo-García, et al., 2006) im Vergleich zu Gesunden, wobei hier vor allem substanzbezogene Reize als problematisch gelten (Su et al., 2017). Auch erhöhtes Delay Discounting konnte bei einer Abhängigkeit von Opioiden (Kirby, Petry \& Bickel, 1999; Robles et al., 2011) und Kokain (Coffey et al., 2003) nachgewiesen werden.

Den Befunden scheinen neurobiologische Veränderungen zugrunde zu liegen, wie sie beispielsweise Li und Sinha (2008) sowie Verdejo-García und Bechara (2009) ausführen. Dies betrifft insbesondere die Hypoaktivität von Arealen, denen reflektierte, kognitive Prozesse zugeschrieben werden, wie die Abwägung langfristiger Konsequenzen. Diese bilden ein top-down-Netzwerk und umfassen beispielsweise den präfrontalen Kortex. Im Zuge der mangelnden Regulation durch das top-down-Netzwerk zeigt sich eine Hyperaktivierung in Arealen, denen automatisierte, reizgesteuerte Reaktionen zugeschrieben werden. Diese bilden ein bottom-up-Netzwerk und umfassen beispielsweise die Amygdala. Dieses Ungleichgewicht der beiden Netzwerke äußert sich unter anderem in impulsiven Reaktionen auf emotionale Belastung, z.B. in Form einer automatisierten Annäherung an stark belohnende Reize, wie die Substanz selbst (Li \& Sinha, 2008; VerdejoGarcía \& Bechara, 2009).

Die Annahme, dass die Regulation von Emotionen im Rahmen einer Substanzabhängigkeit vermehrt über das impulsive System stattfindet, passt zu Befunden im Selbstbericht und auf behavioraler Ebene. So konnte gezeigt werden, dass defizitäre Emotionsregulationskompetenzen mit geringerer Impulskontrolle einhergehen (Fox, Bergquist, Casey, Hong \& Sinha, 2010) und darüber mit pathologischem Alkoholkonsum assoziiert sind (Garofalo \& Velotti, 2015; Jakubczyk et al., 2018). Bei Emotionsregulationskompetenzen handelt es sich um verschiedene Fähigkeiten, die jeweils zu einer effektiven Emotionsregulation beitragen (Berking \& Znoj, 2008).

Ein Ansatzpunkt, um diese automatisierten Prozesse zu durchbrechen, und so eine effektive Emotionsregulation zu fördern und impulsive Verhaltensweisen zu reduzieren, liegt in der Förderung von Achtsamkeit (Mundle, Bowen, Heinz \& Kienast, 2014; Pozuelos, Mead, Rueda \& Malinowski, 2019; Tang, Tang \& Posner, 2016). Darunter versteht man allgemeinhin die Aufmerksamkeitslenkung auf den gegenwärtigen Moment, die mit einer absichtsvollen und nicht-wertenden Haltung verbunden ist, so KabatZinn (2003). Achtsames Verhalten kann also als antagonistisch zu impulsivem bzw. automatisiertem Verhalten verstanden werden (Murphy \& MacKillop, 2012). Die damit verbundene Akzeptanz innerer Zustände, wie beispielsweise emotionaler Spannung (Heidenreich \& Michalak, 2003), scheint eine höhere Emotionstoleranz und einen besseren Umgang mit Craving zu bewirken (Heidenreich, Schneider \& Michalak, 2006). Empirisch gibt es Hinweise darauf, dass der negative Zusammenhang zwischen Achtsamkeit und alkoholbezogenen Problemen über verringertes impulsives Verhalten vermittelt wird (Christopher, Ramsey \& Antick, 2013; Murphy \& MacKillop, 2012). Teper und Inzlicht (2013) fanden ferner bei Gesunden, dass achtsame Meditation die emotionale Akzeptanz und darüber die exekutive Kontrolle verbessert.

Es wird angenommen, dass achtsamkeitsbasierte Techniken die Aktivität des reflektiven Systems stärken und so den impulsiven Umgang mit Emotionen, wie die Annäherung an die stark belohnenden substanzassoziierten Reize verringern (Garland, Froeliger \& Howard, 2014).

So erwiesen sich achtsamkeitsfördernde Therapieprogramme unter anderem als wirksam hinsichtlich einer Reduktion von Craving (Witkiewitz, Bowen, Douglas, \& Hsu, 2013) sowie Rückfallrisiko und -schwere (Bowen et al., 2014).

Insgesamt scheint Achtsamkeit ein wichtiges Korrelat impulsiven Verhaltens bei Substanzkonsumstörungen zu sein, sowohl direkt, als auch vermittelt über eine effektive Emotionsregulation. Einzelne Befunde zu diesen Zusammenhängen liegen in Stichproben mit alkoholabhängigen Patienten vor. Allerdings mangelt es an Befunden zur Wirkbeziehung der drei Konstrukte bei Drogenabhängigkeit.

Dabei ist die vergleichende Betrachtung der verschiedenen Impulsivitäts-Facetten bedeutsam. Bei alkoholabhängigen Patienten zeigte sich beispielsweise kein $\mathrm{Zu}$ sammenhang zwischen Achtsamkeit und impulsivem Entscheidungsverhalten (Murphy \& MacKillop, 2012). Im therapeutischen Kontext (z.B. hinsichtlich Craving und Abstinenzzuversicht) untersuchten bisherige Studien die Bedeutung der Facetten nur isoliert voneinander. So lassen sich für Trait-Impulsivität (Tziortzis, Mahoney, Kalechstein, Newton \& De La Garza, 2011) und Delay Discounting (MacKillop et al., 2010), nicht aber für defizitäre Inhibitionskontrolle (Verdejo-García et al., 2012) positive Zusammenhänge mit Craving erwarten. Abstinenzzuversicht scheint negativ mit Trait-Impulsivität und Delay Discounting zusammenzuhängen (Athamneh et al., 2019; Hayaki et al., 2011). Studien zum Zusammenhang mit Inhibitionskontrolle liegen unseres Wissens nach nicht vor. 
Die vorliegende Pilotstudie untersuchte daher, wie impulsives Verhalten bei drogenabhängigen Patienten in stationärer Therapie mit selbstberichteter Achtsamkeit und Emotionsregulationskompetenzen assoziiert ist, sowie die Bedeutung der verschiedenen Impulsivitäts-Facetten für Craving und Abstinenzzuversicht.

\section{Methodik}

\section{Design}

In einer Querschnittsuntersuchung wurden selbstberichtete und behaviorale Daten erfasst.

\section{Hypothesen}

Es wurde ein direkter, negativer Zusammenhang zwischen selbstberichteter Achtsamkeit und Trait-Impulsivität sowie Defiziten der substanzbezogenen Inhibitionskontrolle erwartet. Ferner wurde angenommen, dass dieser Zusammenhang teilweise über höhere Emotionsregulationskompetenzen mediiert wird.

Darüber hinaus wurde erwartet, dass Trait-Impulsivität und Delay Discounting - bei Berücksichtigung von Inhibitionskontrolle, Achtsamkeit, Emotionsregulationskompetenzen, Depressivität, und Konsumdauer - positiv mit Craving und negativ mit Abstinenzzuversicht in Beziehung stehen.

\section{Setting}

Die Rekrutierung fand in einer stationären Rehabilitationseinrichtung statt. Die dort angebotene 24-wöchige Entwöhnungstherapie richtet sich an erwachsene Männer mit Drogenabhängigkeit ohne akute psychotische Episode.

\section{Stichprobe}

An der Untersuchung nahmen 31 Patienten teil. Die Patienten wurden über Ablauf und Ziele der Studie informiert und willigten schriftlich ein. Sie erhielten keine Kompensation. In den Daten eines Patienten wurden wiederholt Ausreißer identifiziert. Dies wurde - vor dem Hintergrund mangelnder Kooperationsbereitschaft während der Testung - auf nicht-wahrheitsgemäße Antworten zurückgeführt. Somit wurde der Patient von der Datenanalyse ausgeschlossen. Ein positives Ethikvotum der Universität Bamberg liegt vor.

\section{Ein- und Ausschlusskriterien}

Es wurden Patienten ab der vierten Therapiewoche (Ende der Eingewöhnungsphase) eingeschlossen, deren bevorzugte Substanz stimulierende Drogen oder Heroin waren. Zwischen dem letzten Konsum und der Erhebung mussten mindestens vier Wochen liegen. Das selbstberichtete Vorliegen einer diagnostizierten schizophrenieformen Erkrankung führte zum Ausschluss.

\section{Instrumente}

\section{Fragebögen}

Ein Fragebogen zu demographischen Daten enthielt 26 Fragen, unter anderem zu Alter, Bildung, Beginn des Substanzkonsums, Suchtbehandlungen und Konsumhäufigkeit.

Dispositionelle Achtsamkeit wurde durch die deutsche Mindful Attention and Awareness Scale (MAAS; Michalak, Heidenreich, Ströhle \& Nachtigall, 2008) mit einem Gesamtwert zwischen 15 und 90 erhoben (Interne Konsistenz in der vorliegenden Studie $\alpha=.91$ [sehr gut]).

Zur Messung der Emotionsregulationskompetenzen wurde die Trait-Version des Fragebogens zur Selbsteinschätzung emotionaler Kompetenzen (SEK-27; Berking \& Znoj, 2008) gewählt. Nicht für alle Subskalen liegen gute psychometrische Kennwerte vor (Berking \& Znoj, 2008). Aus diesem Grund wurde nur der Gesamtwert genutzt $(\alpha=.95$ [exzellent]), der zwischen 0 und 135 liegt.

Um Trait-Impulsivität zu erfassen, wurde die deutsche Barratt Impulsiveness Scale - Kurzversion mit 15 Items (BIS-15; Meule, Vögele \& Kübler, 2011) gewählt. Nach Empfehlung von Meule et al. (2011) wurde der Gesamtwert betrachtet ( $\alpha=.83$ [gut]), der zwischen 15 und 60 liegt.

Als Maß für Delay Discounting wurde der Delay Discounting Test (DDT) nach Forstmeier und Maercker (2011) verwendet. Die Instruktion wurde dabei in Anlehnung an Kaplan et al. (2016) etwas gekürzt. Beide Autorengruppen (Forstmeier \& Maercker, 2011; Kaplan et al., 2016) orientieren sich an dem originalen Verfahren von Kirby et al. (1999). Berechnet wird die Diskontrate $k$, ein Indikator für die prozentuale Abnahme der empfundenen Belohnung in Abhängigkeit von der Länge des Zeitintervalls und somit für niedrige Selbstkontrolle ( $\alpha=.96$ [exzellent]).

Als Instrument zur substanzunabhängigen Erfassung von Craving wurde die Mannheimer Craving Skala (MaCS; Nakovics, Diehl, Geiselhart \& Mann, 2009) gewählt ( $\alpha=.88$ [gut]), deren Gesamtwert zwischen 0 und 48 liegt. Die vier Zusatzitems wurden in dieser Studie nicht genutzt.

Zur Messung der Abstinenzzuversicht wurde die Kurzversion der Heidelberger Skalen zur Abstinenzzuversicht verwendet (HEISA-16; Körkel \& Schindler, 1998). In dieser Studie wurde der Gesamtwert zwischen 0 und 100, 
nicht aber die einzelnen Subskalen betrachtet $(\alpha=.96$ [exzellent]).

Depressivität wurde mit dem Depressionsmodul des Patient Health Questionnaire (PHQ-9; Löwe, Spitzer, Zipfel \& Herzog, 2002) mit einem Gesamtwert zwischen 0 und 27 erhoben $(\alpha=.79$ [akzeptabel]).

\section{Computergestützte Go-/No-Go-Aufgabe}

Inhibitionskontrolle wurde in Anlehnung an MacKillop et al. (2016) durch eine Go/No-Go-Aufgabe erfasst. Dabei sollte so schnell wie möglich auf einen Go-Reiz (hier in $75 \%$ der Fälle) mit Druck einer Taste reagiert und bei einem NoGo-Reiz die motorische Reaktion unterdrückt werden. Die Errors of Commission (EoCs; fälschlicherweise auf einen No-Go-Reiz reagiert) dienten als Indikator für Defizite der Inhibitionskontrolle (siehe MacKillop et al., 2016). Die Aufgabe wurde mit Python 3.6.6 (Oberfläche PsychoPy 3.2.4, Paket Numpy) programmiert und auf einem Laptopbildschirm (14“) präsentiert. Zu Beginn entschieden sich die Probanden für das Design mit Heroin oder stimulierenden Drogen und bewerteten 15 Gartengegenstände bezüglich deren Assoziation mit dem Substanzkonsum (Skala von 1 bis 10). Die 10 neutralsten Bilder aus diesen wurden automatisiert in die Aufgabe implementiert. Es folgte ein nicht-gewerteter Übungsdurchgang mit geometrischen Formen und Feedback (roter/grüner Bildschirm nach falscher/richtiger Reaktion). Die anschließende Aufgabe bestand aus 3 Teilen mit je 3 Blöcken á 40 Reizen $\left(400^{*} 600 \mathrm{~mm}\right.$, Präsentationsdauer $500 \mathrm{~ms}$, Interstimulusintervall $1000 \mathrm{~ms}$ ). Teil 1 beinhaltete geometrische Formen. In Teil 2 sollte auf Gartengegenstände, nicht aber Substanzen reagiert werden. In Teil 3 war es anders herum. In dieser Studie wurden substanzbezogene Defizite der Inhibitionskontrolle (0 bis 30 EoCs), also Teil 2 analysiert (Heroin: $\alpha=.78$ [akzeptabel], Stimulierende Drogen: $\alpha=.83$ [gut]). Ein exemplarischer Ablauf ist in $\mathrm{Ab}$ bildung 1 dargestellt.

\section{Durchführung}

Die Testung begann mit der Erhebung demographischer und suchtbezogener Charakteristika. Danach wurden die weiteren Tests am Computer in der folgenden Reihenfolge dargeboten: MaCS (Nakovics et al., 2009), HEISA-16 (Körkel \& Schindler, 1998), DDT (Forstmeier \& Maercker, 2011), Go-/No-Go-Aufgabe, BIS-15 (Meule et al., 2011),

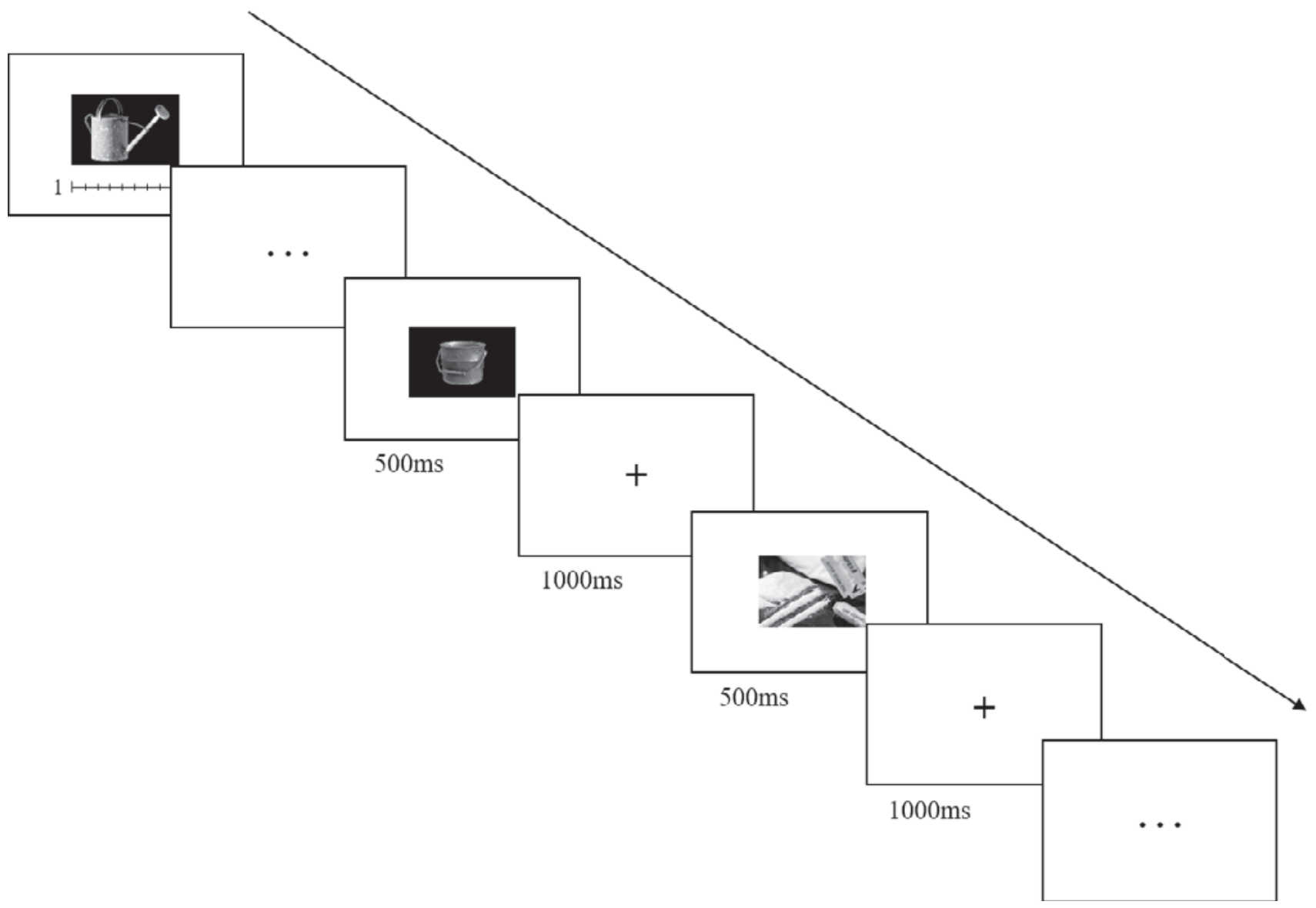

Abbildung 1. Beispielhaftes Rating eines Gartengegenstands und zwei Reize des Designs mit stimulierenden Drogen. 
MAAS (Michalak et al., 2008), SEK-27 (Berking \& Znoj, 2008) und PHQ-9 (Löwe et al., 2002). Die Testdauer betrug ca. 50 Minuten.

\section{Datenanalyse}

Von zwei Probanden fehlten Daten in der Go-/No-GoAufgabe. Alle Analysen wurden folglich per-protocol (listenweiser Fallausschluss) und nach multipler Imputation der fehlenden Werte (Anzahl=10) berechnet. Letzteres wird bei signifikanten Abweichungen von per-protocol berichtet.

Alle Fragestellungen wurden auf einem Signifikanzniveau von $\alpha=5 \%$ untersucht. Der $p$-Wert für mehrfache Gruppenvergleiche wurde nach Bonferroni korrigiert $\left(p_{b}\right)$. Effektstärken werden für signifikante Ergebnisse berichtet.

Eine vorbereitende Analyse zeigte keine Unterschiede (alle $p_{b}=1.0$ ) zwischen Konsumierenden von Heroin und stimulierenden Drogen für MAAS (Heroin: $M=65.64$, $S D=12.22$; Stimulierend: $M=59.89, S D=13.50$ ), SEK-27 (Heroin: $M=66.45, S D=16.04$; Stimulierend: $M=64.63$, $S D=21.88$ ), BIS-15 (Heroin: $M=32.00, S D=7.46$; Stimulierend: $M=33.79, S D=7.08$ ), DDT (Heroin: $M=.06, S D=.09$; Stimulierend: $M=.06, S D=.08$ ), EoCs (Heroin: $M=4.60$, $S D=3.06$; Stimulierend: $M=4.33, S D=3.56$ ), MaCS (Heroin: $M=13.73, S D=7.44$; Stimulierend: $M=15.05, S D=8.76$ ), HEISA-16 (Heroin: $M=63.75, \quad S D=23.24$; Stimulierend: $M=55.92, S D=27.81$ ), PHQ-9 (Heroin: $M=4.45, S D=2.54$; Stimulierend: $M=8.00, S D=5.03$ ) und Konsumdauer (Heroin: $M=23.64, S D=5.33$; Stimulierend: $M=20.00, S D=6.62$ ). Folglich wurden die Gruppen gemeinsam analysiert.

Zur Beantwortung der Fragestellungen wurden in IBM SPSS Statistics 26 multiple lineare Regressionsmodelle und Mediationsanalysen mit der Erweiterung PROCESS v3.4 gerechnet (Hayes, 2018). Bei Letzteren werden die Regressionsgewichte der Pfade und der indirekte Effekt inferenzstatistisch mittels Bootstrapping-Verfahren geschätzt. Der totale Effekt gibt dabei den Einfluss des Prädiktors auf die abhängige Variable an. Der direkte Effekt schätzt ebendiesen Einfluss bei Kontrolle des Mediators. Der indirekte Effekt gibt an, ob der Effekt des Prädiktors auf die abhängige Variable durch den Mediator erklärt werden kann.

\section{Ergebnisse}

\section{Stichprobenbeschreibung}

Die 30 Probanden im Alter von $36.87(S D=6.13)$ Jahren wiesen $11.25(S D=2.49)$ Bildungsjahre auf. Erstkonsum fand im Alter von $15.53(S D=3.44)$ Jahren statt. Neunzehn
Probanden (63\%) nannten stimulierende Drogen und 11 (37\%) Heroin als bevorzugte Substanz. Polyvalent konsumierten 26 (87\%) Probanden. Einundzwanzig (70 \%) Probanden nahmen bereits mindestens einmal an einer Entwöhnungs- und 26 (87\%) an einer Entgiftungsbehandlung teil. Weitere deskriptive Daten sind in Tabelle $1 \mathrm{zu}$ finden.

\section{Zusammenhang zwischen Achtsamkeit und impulsivem Verhalten via Emotionsregulationskompetenzen}

\section{Trait-Impulsivität}

Wie in Abbildung 2a dargestellt, zeigte sich ein signifikanter totaler Effekt $(c)$ von Achtsamkeit (MAAS) auf TraitImpulsivität (BIS-15), sowie ein signifikanter Pfad (a) von Achtsamkeit (MAAS) auf Emotionsregulation (SEK-27). Weitere Pfade wurden nicht signifikant. Es gab keinen signifikanten indirekten Effekt, $B=-0.08, S E(B)=0.09,95 \%$ KI für $B[-0.243,0.116]$.

\section{Defizite der Inhibitionskontrolle}

Wie in Abbildung $2 \mathrm{~b}$ dargestellt, zeigte sich ein signifikanter Pfad ( $a$ ) von Achtsamkeit (MAAS) auf Emotionsregulation (SEK-27). Weitere Pfade wurden nicht signifikant. Es gab einen signifikanten indirekten Effekt von Achtsamkeit (MAAS) auf Inhibitionskontrolle, $B=-0.09, S E(B)=0.05$, $95 \%$ KI für $B$ [-0.212,-0.003]. Der vollständig standardisierte Effekt betrug hier -.34 .

Tabelle 1. Patientencharakteristika und deskriptive Ergebnisse der Testbatterie

\begin{tabular}{llcc}
\hline Kennwert & $n$ & $M$ & \multicolumn{1}{l}{ SD } \\
\hline Konsumdauer in Jahren & 30 & 21.33 & 6.34 \\
HEISA-16 Gesamtwert & 30 & 58.79 & 26.10 \\
MaCS Gesamtwert & 30 & 14.57 & 8.19 \\
DDT Diskontrate k & 30 & .06 & .08 \\
BIS-15 Gesamtwert & 30 & 33.13 & 7.15 \\
MAAS Gesamtwert & 30 & 62.00 & 13.14 \\
SEK-27 Gesamtwert & 30 & 65.30 & 19.66 \\
PHQ-9 Gesamtwert & 30 & 6.70 & 4.58 \\
EoCs Teil 2 & (30) 28 & $(4.42) 4.43$ & 3.34 \\
RT Teil 2 & (30) 28 & .43 & .03 \\
\hline
\end{tabular}

Anmerkungen: Abweichende Werte nach multipler Imputation in Klammern. HEISA-16: Kurzversion der Heidelberger Skalen zur Abstinenzzuversicht. MaCS: Mannheimer Craving Skala. DDT: Delay Discounting Test. BIS-15: Kurzversion der Barratt Impulsiveness Scale. MAAS: Mindful Attention and Awareness Scale. SEK-27: Fragebogen zur Selbsteinschätzung emotionaler Kompetenzen. PHQ-9:Depressionsmodul des Patient Health Questionnaire. EoCs: Errors of Commission. RT: Reaktionszeit in ms. 

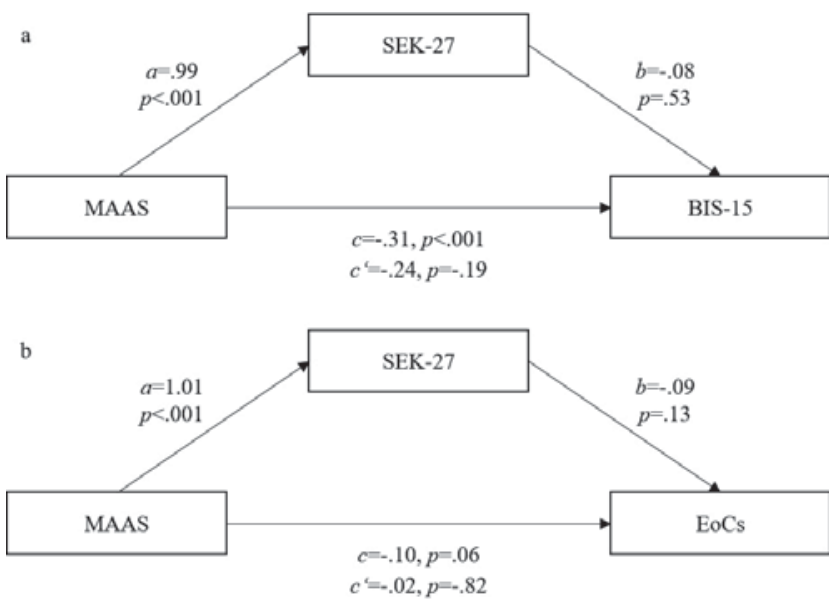

Abbildung 2. Einfache Mediationsmodelle nach Hayes (2018). c: totaler Effekt. c': direkter Effekt. BIS-15: Kurzversion der Barratt Impulsiveness Scale. MAAS: Mindful Attention and Awareness Scale. SEK-27: Fragebogen zur Selbsteinschätzung emotionaler Kompetenzen.

\section{Zusammenhang mit Craving}

Das Modell erwies sich als signifikant, $R^{2}=.55, F(7,20)=3.54$, $p=.012$. Es zeigte sich ein signifikanter Zusammenhang zwischen Trait-Impulsivität (BIS-15) und Craving (MaCS), $B=.96, p=.003, \beta=.83$.

In der Analyse nach multipler Imputation erwies sich auch Delay Discounting (DDT) als signifikant, $B=38.93$, $p_{\text {kombiniert }}=.048$.

\section{Zusammenhang mit Abstinenzzuversicht}

Das Modell erwies sich nicht als signifikant, $R^{2}=.49$, $F(8,19)=2.24, p=.072$. Konsumdauer und Depressivität (PHQ-9) zeigten einen signifikanten Zusammenhang mit Abstinenzzuversicht (HEISA-16), $B=-1.86, p=.042, \beta=-.43$, und $B=-3.15, p=.032, \beta=-.53$.

\section{Diskussion}

Die vorliegende Pilotstudie untersuchte die Bedeutung von selbstberichteter Achtsamkeit und Emotionsregulationskompetenzen für impulsives Verhalten bei drogenabhängigen Patienten. Es wurde erwartet, dass höhere Achtsamkeit, teilweise vermittelt über höhere Emotionsregulationskompetenzen, mit geringerer Trait-Impulsivität und besserer substanzbezogener Inhibitionskontrolle einhergeht.

Selbstberichtete Achtsamkeit war positiv mit den Emotionsregulationskompetenzen assoziiert. Achtsamere Pa- tienten scheinen ihre Emotionen folglich effektiver zu regulieren, wie Heidenreich et al. (2006) annehmen. Ferner fand sich ein totaler, negativer Effekt von Achtsamkeit auf Trait-Impulsivität. Achtsamere Patienten zeigen dem Selbstbericht zufolge also weniger impulsives Verhalten im Alltag. Hier spiegelt sich der von Murphy und MacKillop (2012) postulierte antagonistische Zusammenhang wieder. Dieser wurde allerdings nicht durch bessere Emotionsregulationskompetenz mediiert, zumal sich für Letztere kein Zusammenhang mit Trait-Impulsivität zeigte.

Der indirekte Effekt von selbstberichteter Achtsamkeit auf Defizite der substanzbezogenen Inhibitionskontrolle, in Kombination mit dem fehlenden direkten Effekt, weist auf eine vollständige Mediation hin (Hayes, 2018). Höhere Achtsamkeit scheint folglich mit einer effektiveren Emotionsregulation einherzugehen, welche wiederum mit einer besseren Hemmung der Reaktion auf substanzbezogene Reize assoziiert ist. Dies passt zu den neurobiologischen Befunden, dass Achtsamkeit einen reflektierten Umgang mit Emotionen fördert und so impulsives Verhalten im Rahmen der Emotionsregulation, wie die Annäherung an substanzassoziierte Reize, reduziert (Garland et al., 2014).

Weiterhin wurde die Bedeutung der Impulsivitäts-Facetten für Craving und Abstinenzzuversicht betrachtet. Die Ausprägung des Cravings hing hypothesenkonform mit Trait-Impulsivität und Delay Discounting, nicht aber mit defizitärer Inhibitionskontrolle zusammen. Personen mit höherer Trait-Impulsivität und geringerer Bereitschaft zum Belohnungsaufschub empfanden ein höheres Craving. $\mathrm{Zu}$ beachten ist, dass sich für Delay Discounting nur nach multipler Imputation ein signifikanter positiver Zusammenhang fand (per-protocol knapp nicht signifikant). Dieser Unterschied kann mit erhöhter Power erklärt werden.

Die Ausprägung der Abstinenzzuversicht ließ sich lediglich auf Depressivität und Konsumdauer, nicht aber auf Trait-Impulsivität und Delay Discounting zurückführen. Hypothesenkonform zeigte sich für die Inhibitionskontrolle kein Zusammenhang.

Der Zusammenhang von selbstberichteter Achtsamkeit und effektiver Emotionsregulation sowie Trait-Impulsivität steht jeweils im Einklang mit bisherigen Annahmen (Christopher et al., 2013; Murphy \& MacKillop, 2012; Mundle et al., 2014; Pozuelos et al., 2019; Tang et al., 2016). Es zeigte sich allerdings kein negativer Zusammenhang zwischen effektiver Emotionsregulation und TraitImpulsivität, wie es in bisherigen Studien der Fall war (Fox et al., 2010; Garofalo \& Velotti, 2015; Jakubczyk et al., 2018). Darin wurde Achtsamkeit allerdings nicht berücksichtigt. Zudem handelte es sich um alkoholabhängige Stichproben. Für die Ausprägung impulsiven Verhaltens bei Drogenabhängigkeit scheint also insbesondere Acht- 
samkeit bedeutsam zu sein. Dass diese wiederum vermittelt über eine effektivere Emotionsregulation mit besserer Inhibitionskontrolle zusammenhängt, stützt die Befunde von Teper und Inzlicht (2013) nun auch in einer substanzabhängigen Stichprobe.

Der Zusammenhang zwischen Trait-Impulsivität und Delay Discounting mit Craving steht im Einklang mit bisherigen Befunden und Annahmen (MacKillop et al., 2010; Mohammadzadeh et al., 2018; Tziortzis et al., 2011). Dass selbstberichtete Achtsamkeit und Emotionsregulationskompetenzen nicht mit Craving assoziiert waren, könnte unter anderem daran liegen, dass bestimmte Facetten dieser beiden mehrdimensionalen Konstrukte eine größere Rolle spielen als andere (Garland und Roberts-Lewis, 2013; Ottonello et al., 2019). So scheint für die Reduktion von Craving im Rahmen achtsamkeitsbasierter Interventionen die Achtsamkeitsfacette einer nicht-wertenden Haltung relevant (Witkiewitz, et al., 2013), welche jedoch durch die Erhebungsinstrumente der vorliegenden Studie nicht abgedeckt wurde. Es könnte sein, dass Achtsamkeit im Sinne einer bewussten Wahrnehmung des gegenwärtigen Augenblicks die empfundene Quantität des Cravings zwar steigert, die Bewertung des Cravings im Sinne einer nicht-wertenden Akzeptanz allerdings funktionaler ist, was Dauer und Belastung des Cravings reduziert. Zudem wurden die Zusammenhänge bisher stets isoliert von Impulsivität betrachtet.

Dass sich kein Zusammenhang zwischen Trait-Impulsivität und Delay Discounting auf die Abstinenzzuversicht zeigte, passt ebenfalls nicht zu bestehenden Befunden (Athamneh et al., 2019; Hayaki et al., 2011). Die Unterschiede könnten durch abweichende Stichprobenzusammensetzungen im Hinblick auf die konsumierte Substanz erklärt werden. Stattdessen erwiesen sich Konsumdauer und Depressivität als signifikante Prädiktoren. Dies stützt die bisherige Forschung (Dolan, Martin \& Rohsenow, 2008) und ist ein wichtiger Befund für künftige Studien.

Die vorliegende Arbeit weist durchaus Kritikpunkte auf. Dazu gehört die kleine Stichprobengröße und die Untersuchung ausschließlich männlicher Probanden. Da eine gesunde Kontrollgruppe fehlte, kann auch keine Aussage darübergetroffen werden, ob die gefundenenZusammenhänge spezifisch für eine drogenabhängige Stichprobe sind. Weiterhin lassen sich die Ergebnisse nicht ohne weiteres auf andere Patientengruppen, z. B. weibliche Patientinnen generalisieren, auch wenn die externe Validität der Stichprobenzusammensetzung insgesamt hoch ist. Ferner sind aufgrund des Designs keine Aussagen zu Kausalitäten bzw. zur Wirkrichtung möglich. So könnten impulsive Verhaltensweisen auch aus erhöhtem Craving resultieren, anstatt dieses zu beeinflussen (Mundle et al., 2014). Auch wurde die Schwere der Abhängigkeit in der vorliegenden Studie nicht berücksichtigt, die jedoch einen wesentlichen Einfluss auf die gefundenen Zusammenhänge haben könnte.

Dennoch ist die vorliegende Pilotstudie nach unserem Wissen die Erste, die sich mit den Zusammenhängen zwischen impulsivem Verhalten, selbstberichteter Achtsamkeit und Emotionsregulationskompetenzen bei Drogenabhängigkeit beschäftigt sowie die Bedeutung verschiedener Impulsivitäts-Facetten für Craving und Abstinenzzuversicht parallel beleuchtet. Sie zeichnet sich insbesondere durch die Individualisierung der Go-/No-Go-Aufgabe aus.

Es ergeben sich Implikationen für weitere Forschung. So wäre es z.B. wichtig, Unterschiede zu einer gesunden Stichprobe oder zwischen Substichproben (Substanzarten, Geschlechter) zu untersuchen sowie Achtsamkeit, Emotionsregulationskompetenzen und Trait-Impulsivität als mehrdimensionale Konstrukte (Berking \& Znoj, 2008; MacKillop et al., 2016; Michalak et al., 2016) zu erfassen, also die Bedeutung verschiedener Facetten zu untersuchen. $\mathrm{Zu}$ beachten ist in diesem Zusammenhang auch, dass - mit Ausnahme der Inhibitionskontrolle - alle Konstrukte nur im Selbstbericht erhoben wurden.

Um die gefundenen Zusammenhänge hinsichtlich ihrer Kausalität zu prüfen, sind experimentelle Designs nötig.

Zusammenfassend sprechen die Befunde für die Differenzierung verschiedener Facetten von Impulsivität (MacKillop et al., 2016). So scheint die Ausprägung der TraitImpulsivität vor allem mit geringerer, selbstberichteter Achtsamkeit assoziiert zu sein und gemeinsam mit Defiziten im Belohnungsaufschub eine besondere Rolle für Craving zu spielen. Für Abstinenzzuversicht hingegen hat Impulsivität eher keine Relevanz. Bedeutender ist in diesem Fall die Ausprägung der Depressivität. Auch für die Inhibitionskontrolle erwies sich Achtsamkeit als wichtiges positives Korrelat. Der Zusammenhang wurde hier durch effektivere Emotionsregulation vermittelt. Es wäre nun interessant, die Bedeutung von Impulsivität als Wirkvariable bei achtsamkeitsbasierter Therapieansätzen (Bowen et al., 2014; Witkiewitz et al., 2013) zu untersuchen.

\section{Perspektiven für die klinische Praxis}

- Die vorliegende Pilotstudie weist auf einen bedeutsamen Zusammenhang zwischen Trait-Impulsivität und Craving während einer stationären Entwöhnungstherapie hin.

- Ferner scheint selbstberichtete Achtsamkeit wesentlich mit Impulskontrolle assoziiert zu sein.

- Künftige Studien sollten Achtsamkeit als Ansatzpunkt zur Reduktion impulsiven Verhaltens in experimentellen und Interventionsstudien untersuchen. 


\section{Literatur}

Athamneh, L. N., DeHart, W.B., Pope, D., Mellis, A.M., Snider, S.E., Kaplan, B.A. et al. (2019). The phenotype of recovery III: Delay discounting predicts abstinence self-efficacy among individuals in recovery from substance use disorders. Psychology of Addictive Behaviors, 33, 310-317.

Berking, M. \& Znoj, H. (2008). Entwicklung und Validierung eines Fragebogens zur standardisierten Selbsteinschätzung emotionaler Kompetenzen (SEK-27). Zeitschrift für Psychiatrie, Psychologie und Psychotherapie, 56, 141-153.

Bowen, S., Witkiewitz, K., Clifasefi, S. L., Grow, J., Chawla, N., Hsu, S.H. et al. (2014). Relative efficacy of mindfulness-based relapse prevention, standard relapse prevention, and treatment as usual for substance use disorders: a randomized clinical trial. JAMA Psychiatry, 71(5), 547-556.

Christopher, M., Ramsey, M. \& Antick, J. (2013). The role of dispositional mindfulness in mitigating the impact of stress and impulsivity on alcohol-related problems. Addiction Research and Theory, 21, 429-434.

Coffey, S. F., Gudleski, G. D., Saladin, M.E. \& Brady, K.T. (2003). Impulsivity and Rapid Discounting of Delayed Hypothetical Rewards in Cocaine-Dependent Individuals. Experimental and Clinical Psychopharmacology, 11, 18-25.

Dolan, S. L., Martin, R.A. \& Rohsenow, D.J. (2008). Self-Efficacy for Cocaine Abstinence: Pretreatment Correlates and Relationship to Outcomes. Addictive Behavior, 33, 675-688.

Fernández-Serrano, M.J., Perales, J.C., Moreno-López, L., PérezGarcía, M. \& Verdejo-García, A. (2012). Neuropsychological profiling of impulsivity and compulsivity in cocaine dependent individuals. Psychopharmacology, 219,673-683.

Forstmeier, S. \& Maercker, A. (2011). Selbstkontrolle im höheren Erwachsenenalter: Eine deutsche Version des Delay Discounting Tests von Kirby. Psychotherapie Psychosomatik Medizinische Psychologie, 61, e19-e33.

Fox, H.C., Bergquist, K. L., Casey, J., Hong, K.A. \& Sinha, R. (2010). Selective Cocaine-Related Difficulties in Emotional Intelligence: Relationship to Stress and Impulse Control. American Journal on Addictions, 20,151-160.

Garland, E., Froeliger, B. \& Howard, M. (2014). Mindfulness training targets neurocognitive mechanisms of addiction at the attention-appraisal-emotion interface. Frontiers in psychiatry, 4,173.

Garland, E. \& Roberts-Lewis, A. (2013). Differential Roles of Thought Suppression and Dispositional Mindfulness in Porsttraumatic Stress Symptoms and Craving. Addictive Behaviors, 38, 1555-1562.

Garofalo, C. \& Velotti, P. (2015). Alcohol misuse in psychiatric patients and nonclinical individuals: The role of emotion dysregulation and impulsivity. Addiction Research \& Theory, 23, 294-300.

Hayaki, J., Herman, D.S., Hagerty, C.E., de Dios, M.A., Anderson, B.J. \& Stein, M.D. (2011). Expectancies and Self-Efficacy Mediate the Effects of Impulsivity on Marijuana Use Outcomes: An Application of the Acquired Preparedness Model. Addictive Behaviors, 36, 389-396.

Hayes, A. F. (2018). Introduction to Mediation, Moderation, and Conditional Process Analysis. A Regression-Based Approach (2nd ed.). Guilford Press: New York.

Heidenreich, T., \& Michalak, J. (2003). Achtsamkeit («Mindfulness») als Therapieprinzip in Verhaltenstherapie und Verhaltensmedizin. Verhaltenstherapie, 13(4), 264-274.

Heidenreich, T., Schneider, R., \& Michalak, J. (2006). Achtsamkeit: Ein neuer Ansatz zur Psychotherapie süchtigen Verhaltens. SUCHT, 52(2), 140-149.

Jakubczyk, A., Trucco, E.M., Kopera, M., Kobyliński, P., Suszek, H., Fudalej, S. et al. (2018). The association between impulsivity, emotion regulation, and symptoms of alcohol use disorder. Journal of Substance Abuse Treatment, 91, 49-56.

Kabat-Zinn, J. (2003). Mindfulness-based interventions in context: past, present, and future. Clinical Psychology: Science and Practice, 10(2), 144-156.

Kaplan, B.A., Amlung, M., Reed, D.D., Jarmolowicz, D.P., McKerchar, T.L. \& Lemley, S.M. (2016). Automating scoring of delay discounting for the 21-and 27-item monetary choice questionnaires. The Behavior Analyst, 39(2), 293-304.

Kirby, K. N., Petry, N. M. \& Bickel, W. K. (1999). Heroin Addicts Have Higher Discount Rates for Delayed Rewards Than Non-DrugUsing Controls. Journal of Experimental Psychology, 128 78-87.

Körkel, J. \& Schindler, C. (1998). Die Heidelberger Skalen zur Abstinenzzuversicht (HEISA-16 und HEISA-38). In A. Glöckner, F. Rist \& H. Küfner (Hrsg.), Elektronisches Handbuch zu Erhebungsinstrumenten im Suchtbereich (EHES). Version 2.00. Mannheim: Zentrum für Umfragen, Methoden und Analysen.

Li, C.S.R., \& Sinha, R. (2008). Inhibitory control and emotional stress regulation: Neuroimaging evidence for frontal-limbic dysfunction in psycho-stimulant addiction. Neuroscience \& Biobehavioral Reviews, 32(3), 581-597.

Löwe, B., Spitzer, R. L., Zipfel, S. \& Herzog, W. (2002). Gesundheitsfragebogen für Patienten (PHQ-D). Manual und Testunterlagen (2. Auflage). Karlsruhe: Pfizer.

MacKillop, J., Miranda, R., Monti, P. M., Ray, L.A., Murphy, J. G., Rohsenow, D.J. et al. (2010). Alcohol Demand, Delayed Reward Discounting, and Craving in relation to Drinking and Alcohol Use Disorders. Journal of Abnormal Psychology, 119, 106-114.

MacKillop, J., Weafer, J., Gray, J.C., Oshri, A., Palmer, A. \& de Wit, H. (2016). The latent structure of impulsivity: impulsive choice, impulsive action, and impulsive personality traits. Psychopharmacology, 233, 3361-3370.

Meule, A., Vögele, C. \& Kübler, A. (2011). Psychometrische Evaluation der deutschen Barratt Impulsiveness Scale - Kurzversion (BIS-15). Diagnostica, 57, 126-133.

Michalak, J., Heidenreich, T., Ströhle, G. \& Nachtigall, C. (2008). Die deutsche Version der Mindful Attention and Awareness Scale (MAAS). Psychometrische Befunde zu einem Achtsamkeitsfragebogen. Zeitschrift für Klinische Psychologie und Psychotherapie, 37, 200-208.

Michalak, J., Zarbock, G., Drews, M., Otto, D., Mertens, D., Ströhle, G. et al. (2016). Erfassung von Achtsamkeit mit der deutschen Version des Five Facet Mindfulness Questionnaires (FFMQ-D). Zeitschrift für Gesund heitspsychologie, 24(1), 1-12.

Moeller, F.G., Barratt, E.S., Dougherty, D.M., Schmitz, J.M., \& Swann, A.C. (2001). Psychiatric aspects of impulsivity. American Journal of Psychiatry, 158(11), 1783-1793.

Mohammadzadeh, A., Khosravani, V. \& Feizi, R. (2018). The comparison of impulsivity and craving in stimulant-dependent, opiate-dependent and normal individuals. Journal of Substance Use, 23, 312-317.

Mundle, G., Bowen, S., Heinz, A. \& Kienast, T. (2014). Praktische Anwendung von Achtsamkeit in der Suchttherapie am Beispiel des MBRP Programms und der DBT-Sucht. SUCHT, 60 29-36.

Murphy, C. \& MacKillop, J. (2012). Living in the here and now: interrelationships between impulsivity, mindfulness, and alcohol misuse. Psychopharmacology, 219, 527-536.

Nakovics, H., Diehl, A., Geiselhart, H. \& Mann, K. (2009). Entwicklung und Validierung eines Instruments zur substanzunabhängigen Erfassung von Craving: Die Mannheimer Craving Scale (MaCS). Psychiatrische Praxis, 36, 72-78.

Ottonello, M., Fiabane, E., Pistarini, C., Spigno, P. \& Torselli, E. (2019). Difficulties In Emotion Regulation During Rehabilitation For Alcohol Addiction: Correlations With Metacognitive Beliefs 
About Alcohol Use And Relapse Risk. Neuropsychiatric Disease and Treatment, 15, 2917-2925.

Pozuelos, J.P., Mead, B.R., Rueda, M.R. \& Malinowski, P. (2019). Short-term mindful breath awareness training improves inhibitory control and response monitoring. Progress in Brain Research, 244, 137-163.

Robles, E., Huang, B.E., Simpson, P.M. \& McMillan, D.E. (2011). Delay discounting, impulsiveness, and addiction severity in opioid-dependent patients. Journal of Substance Abuse Treatment, 41, 354-362.

Schultz, J., Neumann, R., \& Steins-Loeber, S. (2019). Impulsivity and relapse during treatment of methamphetamine use disorder. SUCHT, 65, 263-270.

Su, B., Yang, L., Wang, G.Y., Wang, S., Li, S., Cao, H. \& Zhang, Y. (2017). Effect of drug-related cues on response inhibition through abstinence: A pilot study in male heroin abstainers. The American journal of drug and alcohol abuse, 43, 664-670.

Tang, Y.-Y., Tang, R. \& Posner, M.I. (2016). Mindfulness meditation improves emotion regulation and reduces drug abuse. Drug and Alcohol Dependence, 163,13-18.

Taylor, E. M., Murphy, A., Boyapati, V., Ersche, K. D., Flechais, R., Kuchibatla, S. et al. (2016). Impulsivity in abstinent alcohol and polydrug dependence: a multidimensional approach. Psychopharmacology, 233, 1487-1499.

Teper, R. \& Inzlicht, M. (2013). Meditation, mindfulness and executive control: the importance of emotional acceptance and brain-based performance monitoring. Social Cognitive and Affective Neuroscience, 8, 85-92.

Tziortzis, D., Mahoney, J.J., Kalechstein, A. D., Newton, T.F. \& De La Garza, R. (2011). The relationship between impulsivity and craving in cocaine- and methamphetamine-dependent volunteers. Pharmacology, Biochemistry and Behavior, 98, 196-202.

Verdejo-García, A., \& Bechara, A. (2009). A somatic marker theory of addiction. Neuropharmacology, 56, 48-62.

Verdejo-García, A., Bechara, A., Recknor, E.C., \& Perez-Garcia, M. (2006). Executive dysfunction in substance dependent individuals during drug use and abstinence: an examination of the behavioral, cognitive and emotional correlates of addiction. Journal of the International Neuropsychological Society, 12, 405-415.
Verdejo-García, A., Lubman, D.i., Schwerk, A., Roffel, K., Vilar-López, R., MacKenzie, T. et al. (2012). Effect of craving induction on inhibitory control in opiate dependence. Psychopharmacology, 219, 519-526.

Witkiewitz, K., Bowen, S., Douglas, H., \& Hsu, S. H. (2013). Mindfulness-based relapse prevention for substance craving. Addictive Behaviors, 38(2), 1563-1571.

\section{Historie}

Manuskript eingereicht: 30.06 .2020

Manuskript angenommen: 19.02.2021

\section{Danksagung}

Wir danken der Fachklinik Aggerblick Overath für die Unterstützung bei der Rekrutierung der Probanden.

\section{Deklaration möglicher konkurrierender Interessen}

Keine

\section{Förderung}

Open-Access-Veröffentlichung ermöglicht durch die Otto-Friedrich-Universität Bamberg.

\section{ORCID}

Daniela Reichl

(D) https://orcid.org/0000-0002-4696-7001

\section{Daniela Reichl, M. Sc.}

Lehrstuhl für Klinische Psychologie und Psychotherapie Otto-Friedrich-Universität Bamberg

Markusplatz 3

96047 Bamberg

Deutschland

daniela.reichl@uni-bamberg.de 